\title{
EXISTENCE OF SOLUTIONS TO THE THREE DIMENSIONAL BAROTROPIC-VORTICITY EQUATION*
}

\author{
B. EMAMIZADEH ${ }^{\dagger}$ AND M. H. MEHRABI ${ }^{\ddagger}$
}

\begin{abstract}
We prove existence of maximizers for a variational problem in $\mathbb{R}_{+}^{3}$. Solutions represent steady geophysical flows over a surface of variable height which is bounded from below.
\end{abstract}

Key words. Rearrangements, Vortices, Variational problems, Barotropic vorticity equation

AMS subject classifications. 35J60, 76B03, 76B47, 76M30, 76U05

1. Introduction. In this paper we prove existence of maximizers for a variational problem which describes a geophysical flow over a surface of variable height, bounded from below, such as a seamount in the ocean or a mountain in the atmosphere. The basic equation governing such flows is the three dimensional barotropic vorticity equation given by

$$
[\psi, \zeta]=0
$$

where [., .] denotes the Jacobian and $\psi$ represents the stream function, $-\zeta$ the potential vorticity given by

$$
-\zeta=\Delta \psi+h,
$$

where $h$ is the height of the bottom surface.

In [6] and [9] similar problems have been considered in two dimensions. Here, the problem has been formulated in three dimensions which is more realistic. In addition, from a technical point of view, due to drastic differences between the fundamental solutions of $-\Delta$ in two and three dimensions the estimates in [6] and [9] are not applicable. In particular we single out the simple but crucial result stated in Lemma 6 in section 3 .

To prove the existence we follow the method proposed by Benjamin [3]. To do this we begin by considering the variational problem over half spheres. In order to prove existence of maximizers in this situation we employ the technology extensively developed by Burton $[4,5]$. Then using a limiting argument we show that maximizers for large half spheres indeed are maximizers for the original problem; the radius of the critical half sphere turns out to be the radius of the smallest two dimensional disc containing the support of the height function $h$.

2. Definitions and notations. Henceforth we assume $p \in(3, \infty)$. The ball centered at $x \in \mathbb{R}^{3}$ with radius $R$ is denoted $B_{R}(x)$; in particular when the center is the origin we write $B_{R}$. For $x=\left(x_{1}, x_{2}, x_{3}\right) \in \mathbb{R}^{3}$, we write $\bar{x}=\left(x_{1}, x_{2},-x_{3}\right)$ and we define $\mathbb{R}_{+}^{3}=\left\{x \in \mathbb{R}^{3}: x_{3}>0\right\}$. For a measurable set $A \subseteq \mathbb{R}^{3},|A|$ denotes the three dimensional Lebesgue measure of $A$. If $A$ is measurable, then $x \in A$ is called a density point of $A$ whenever $\left|B_{\varepsilon}(x) \bigcap A\right|>0$, for all positive $\varepsilon$. The set of all density

\footnotetext{
*Received June 28, 2005; accepted for publication November 21, 2007.

${ }^{\dagger}$ The Petroleum Institute, Department of Mathematics, P.O. Box 2533, Abu Dhabi, United Arab Emirates (bemamizadeh@pi.ac.ae). Corresponding author.

${ }^{\ddagger}$ Department of Mathematics, Iran University of Science and Technology, Narmak, Tehran, Iran (m_h_mehrabi@iust.ac.ir).
} 
points of $A$ is denoted $\operatorname{den}(A)$.

For a measurable function $\zeta: \mathbb{R}_{+}^{3} \rightarrow \mathbb{R}$, the strong support or simply the support of $\zeta$ denoted $\operatorname{supp}(\zeta)$ is defined

$$
\operatorname{supp}(\zeta)=\left\{x \in \mathbb{R}_{+}^{3}: \zeta(x)>0\right\} .
$$

If $f$ and $g$ are non-negative measurable functions that vanish outside sets of finite measure in $\mathbb{R}_{+}^{3}$, we say $f$ is a rearrangement of $g$ whenever

$$
\left|\left\{x \in \mathbb{R}_{+}^{3}: f(x) \geq \alpha\right\}\right|=\left|\left\{x \in \mathbb{R}_{+}^{3}: g(x) \geq \alpha\right\}\right|,
$$

for every positive $\alpha$. Let us fix $\zeta_{0} \in L^{p}\left(\mathbb{R}_{+}^{3}\right)$ to be a non-negative function vanishing outside a set of measure $\frac{4}{3} \pi a^{3}$ for some positive $a$ and $\left\|\zeta_{0}\right\|_{p}=1$. The set of all rearrangements of $\zeta_{0}$ on $\mathbb{R}_{+}^{3}$ which vanish outside bounded sets is denoted $\mathcal{F}$. The subset of $\mathcal{F}$ containing functions vanishing outside the ball $B_{R}$ is denoted $\mathcal{F}(R)$; henceforth we assume $R>a$ in order to ensure $\mathcal{F}(R)$ is non-empty. For a nonnegative $\zeta \in L^{p}\left(\mathbb{R}_{+}^{3}\right)$ having bounded support, we define the energy functional

$$
\Psi(\zeta)=\frac{1}{2} \int_{\mathbb{R}_{+}^{3}} \zeta K \zeta+\int_{\mathbb{R}_{+}^{3}} \eta \zeta
$$

where

$$
K \zeta(x)=\frac{1}{4 \pi} \int_{\mathbb{R}_{+}^{3}}\left(\frac{1}{|x-y|}+\frac{1}{|x-\bar{y}|}\right) \zeta(y) d y
$$

and

$$
\eta(x)=\frac{1}{2 \pi} \int_{\partial \mathbb{R}_{+}^{3}} \frac{1}{|x-y|} h(y) d \sigma(y) .
$$

Here $h \in L^{p}\left(\partial \mathbb{R}_{+}^{3}\right)$ is a non-negative function with compact support.

Let $B_{r_{h}}$ be the smallest ball containing $\operatorname{supp}(h)$; we assume that

$$
r_{h}>\max \left\{a, r_{*}\right\}
$$

where

$$
r_{*} \ln \frac{r_{*}}{2 \sqrt{e}}=2,
$$

(such $r_{*}$ is unique and $1.81 e<r_{*}<1.82 e$ ) and

$$
h\left(x_{1}, x_{2}\right) \geq c \ln \left|x_{1} x_{2}\right|,
$$

almost everywhere in $\operatorname{supp}(h)$, where $c$ is some constant given in Lemma 1.

Let us now introduce the following variational problem $(\mathrm{P})$ :

$$
\sup _{\zeta \in \mathcal{F}} \Psi(\zeta) \text {. }
$$

The solution set for $(P)$ is denoted $\Sigma$. Now we can state the main result of this paper is the following

Theorem. The variational problem $(P)$ is solvable; that is, $\Sigma$ is not empty. Moreover, if $\hat{\zeta} \in \Sigma$ and we set $\hat{\psi}=K \hat{\zeta}+\eta$, then $\hat{\psi}$ satisfies the following partial differential equation

$$
-\Delta \hat{\psi}=\phi \circ \hat{\psi}+h
$$

almost everywhere in $\mathbb{R}_{+}^{3}$, for some increasing function $\phi$ unknown a priori. 
3. Preliminaries. In this section we present some lemmas which will be used in the proof of the Theorem. Then

Lemma 1. Suppose $\zeta \in L^{p}\left(\mathbb{R}_{+}^{3}\right)$ is a non-negative function with compact support.

$$
K \zeta(x) \leq c\|\zeta\|_{p}, \forall x \in \mathbb{R}_{+}^{3} .
$$

Proof. We have

$$
K \zeta(x) \leq \frac{1}{2 \pi} \int_{\mathbb{R}_{+}^{3}} \frac{\zeta(y)}{|x-y|} d y \leq \frac{1}{2 \pi} \int_{B_{r^{*}}(x)} \frac{\tilde{\zeta}(y)}{|x-y|} d y,
$$

where $\tilde{\zeta}$ is the Schwarz rearrangement of $\zeta$ with respect to $x$ and $r^{*}=\left(\frac{3|\operatorname{supp}(\zeta)|}{4 \pi}\right)^{\frac{1}{3}}$. The second inequality is a consequence of Hardy-Littlewood inequality [8]. Now by Hölder's inequality, we get (4), where

$$
c=\frac{1}{2 \pi}\left(\int_{B_{r^{*}}(x)} \frac{1}{|x-y|^{p^{\prime}}} d y\right)^{\frac{1}{p^{\prime}}}=\frac{2(3|\operatorname{supp}(\zeta)|)^{\frac{1}{p^{\prime}}-\frac{1}{3}}}{(4 \pi)^{\frac{2}{3}}\left(3-p^{\prime}\right)^{\frac{1}{p^{\prime}}}},
$$

and $p$ is the conjugate exponent of $p$.

Remark. The constant $c$ evaluated in (5) is the constant used in (2).

Lemma 2. Let $q \geq 1$ and let $U$ be a bounded open subset of $\mathbb{R}_{+}^{3}$. Then $K: L^{p}(U) \rightarrow L^{q}(U)$ is a compact linear operator. Moreover, if $\zeta \in L^{p}\left(\mathbb{R}_{+}^{3}\right)$ vanishes outside $U$, then $K \zeta \in W_{\text {loc }}^{2, p}\left(\mathbb{R}_{+}^{3}\right)$ and verifies

$$
-\Delta u=\zeta \text { a.e in } \mathbb{R}_{+}^{3}
$$

and

$$
\frac{\partial u}{\partial x_{3}}=0 \text { on } \partial \mathbb{R}_{+}^{3}
$$

Proof. From Lemma 1, it readily follows that the map $K$ from $L^{p}(U)$ into $L^{q}(U)$ is well defined. Notice that functions in $L^{p}(U)$ are interpreted as functions in $L^{p}\left(\mathbb{R}_{+}^{3}\right)$ which vanish outside $U$. Now consider $\zeta \in L^{p}\left(\mathbb{R}_{+}^{3}\right)$, which vanishes outside $U$. Then there exists a sequence $\left\{\zeta_{n}\right\}$ in $C_{0}^{\infty}\left(\mathbb{R}_{+}^{3}\right)$ such that $\operatorname{supp}\left(\zeta_{n}\right) \subseteq U$, and $\zeta_{n} \rightarrow \zeta$ in $L^{p}\left(\mathbb{R}_{+}^{3}\right)$, as $n \rightarrow \infty$. We deduce from (4) that

$$
\left|K\left(\zeta_{n}-\zeta\right)(x)\right| \leq c\left\|\zeta_{n}-\zeta\right\|_{p} .
$$

Therefore, $K \zeta_{n} \rightarrow K \zeta$, uniformly in $\mathbb{R}_{+}^{3}$. Whence

$$
\int\left(K \zeta_{n}\right) \phi \rightarrow \int(K \zeta) \phi \quad \forall \phi \in C_{0}^{\infty}\left(\mathbb{R}_{+}^{3}\right) .
$$


On the other hand by $[7$, lemmas $4.1,4.2]$, we have

$$
-\Delta\left(K \zeta_{n}\right)=\zeta_{n} .
$$

Thus by applying the Lebesgue dominated convergence theorem, we infer

$$
\int-\Delta\left(K \zeta_{n}\right) \phi \rightarrow \int \zeta \phi
$$

Note that,

$$
\int-\Delta\left(K \zeta_{n}\right) \phi=\int\left(K \zeta_{n}\right)(-\Delta \phi) .
$$

From (6) we now deduce

$$
\int-\Delta\left(K \zeta_{n}\right) \phi \rightarrow \int(K \zeta)(-\Delta \phi) .
$$

Hence, from (7) and (8), we find

$$
\int \zeta \phi=\int(K \zeta)(-\Delta \phi),
$$

so

$$
-\Delta(K \zeta)=\zeta \quad \text { in } \quad \mathcal{D}^{\prime}\left(\mathbb{R}_{+}^{3}\right) .
$$

Now, by Agmon's regularity theory [2, theorem 6.1] we infer that $K \zeta \in W_{\text {loc }}^{2, p}\left(\mathbb{R}_{+}^{3}\right)$. Therefore equation (9) holds almost everywhere in $\mathbb{R}_{+}^{3}$. According to Sobolev embedding theorem [1] in order to show compactness of $K$ it suffices to prove the boundedness of $K$ as a map from $L^{p}(U)$ into $W^{1,3}(U)$. To do this, we first show

$$
|\nabla K \zeta(x)| \leq M\|\zeta\|_{p} \quad \forall x \in \mathbb{R}_{+}^{3},
$$

where $M$ is a constant independent of $x$.

We begin with

$$
\nabla K \zeta(x)=-\frac{1}{4 \pi} \int_{\mathbb{R}_{+}^{3}}\left(\frac{x-y}{|x-y|^{3}}+\frac{x-\bar{y}}{|x-\bar{y}|^{3}}\right) \zeta(y) d y .
$$

Therefore

$$
\begin{aligned}
|\nabla K \zeta(x)| \leq & \frac{1}{4 \pi} \int_{\mathbb{R}_{+}^{3}}\left(\frac{1}{|x-y|^{2}}+\frac{1}{|x-\bar{y}|^{2}}\right) \zeta(y) d y \\
& \leq \frac{1}{2 \pi} \int_{\mathbb{R}_{+}^{3}} \frac{1}{|x-y|^{2}} \zeta(y) d y \\
& \leq \frac{1}{2 \pi} \int_{B_{r^{*}}(x)} \frac{1}{|x-y|^{2}} \tilde{\zeta}(y) d y
\end{aligned}
$$


where $\tilde{\zeta}$ and $r^{*}$ are the same as in the proof of Lemma 1 .

Now, by Hölder's inequality, we obtain (10), where

$$
M=\frac{2(3|\operatorname{supp}(\zeta)|)^{\frac{1}{p^{\prime}}-\frac{2}{3}}}{\left.(4 \pi)^{\frac{1}{3}}(3-2 p)^{\prime}\right)^{\frac{1}{p^{\prime}}}} .
$$

This implies that

$$
\|\nabla K \zeta\|_{L^{3}(U)} \leq M\|\zeta\|_{p}|U|^{\frac{1}{3}} .
$$

Also, from (4), we have

$$
\|K \zeta\|_{L^{3}(U)} \leq c\|\zeta\|_{p}|U|^{\frac{1}{3}}
$$

Therefore

$$
\|K \zeta\|_{W^{1,3}(U)} \leq \mathcal{C}\|\zeta\|_{p} .
$$

So $K$ is bounded as desired. Finally, by calculation, we have

$$
\frac{\partial K \zeta}{\partial x_{3}}=0 \quad \text { on } \quad \partial \mathbb{R}_{+}^{3},
$$

as desired. $\mathrm{Q}$

The next lemma has been proved in [4].

LEMma 3. If $\overline{\mathcal{F}(R)^{\omega}}$ denotes the weak closure of $\mathcal{F}(R)$ in $L^{p}\left(B_{R}\right)$, then $\overline{\mathcal{F}(R)^{\omega}}$ is convex and weakly sequentially compact.

In order to prove the existence part of the Theorem, we first consider the following truncated variational problem $\left(P_{R}\right)$ :

$$
\sup _{\zeta \in \mathcal{F}(R)} \Psi(\zeta)
$$

We denote the solution set of $\left(P_{R}\right)$ by $\Sigma_{R}$. We show that $\left(P_{R}\right)$ is solvable. To do this we need the following result, which is a simple variation of [5, Lemma 2.15].

Lemma 4 Let $g \in L^{p \prime}\left(B_{R}\right)$ and denote by $L_{\alpha}(g)$ the level set of $g$ at height $\alpha$; that is,

$$
L_{\alpha}(g)=\left\{x \in B_{R}: g(x)=\alpha\right\} .
$$

Let $\mathcal{I}: L^{p}\left(B_{R}\right) \rightarrow \mathbb{R}$ be the linear functional defined by

$$
\mathcal{I}(\zeta)=\int_{B_{R}} \zeta g
$$

If $\hat{\zeta}$ is a maximizer of $\mathcal{I}$ relative to $\overline{\mathcal{F}(R)^{\omega}}$ and if

$$
\left|L_{\alpha}(g) \cap \operatorname{supp}(\hat{\zeta})\right|=0,
$$

for every $\alpha \in \mathbb{R}$, then $\hat{\zeta} \in \mathcal{F}(R)$ and

$$
\hat{\zeta}=\phi_{R} \circ g,
$$


almost everywhere in $B_{R}$, for some increasing function $\phi_{R}$.

Remark. In Lemma 4, by redefining $\hat{\zeta}$ on a set of zero measure on $B_{R}$, if necessary, we can make the conclusion to hold everywhere in $B_{R}$.

Lemma 5. The variational problem $\left(P_{R}\right)$ is solvable. Moreover if $\hat{\zeta}_{R} \in \Sigma_{R}$, then

$$
\hat{\zeta}_{R}=\phi_{R} \circ\left(K \hat{\zeta}_{R}+\eta\right),
$$

almost everywhere in $B_{R}$ for some increasing function $\phi_{R}$.

Proof. By Lemma 2 we have $-\Delta \eta=h$; hence using elliptic regularity theory it follows that $\eta \in W_{\text {loc }}^{2, p}\left(\mathbb{R}_{+}^{3}\right)$, thus $\eta \in C\left(\mathbb{R}_{+}^{3}\right)$, by the Sobolev embedding theorem. Note that $\Psi$ is the summation of a quadratic and a linear functional; that is, $\Psi=Q+\mathcal{L}$. By Lemma $2, Q$ is weakly sequentially continuous. Also since $\eta$ is continuous, it follows that $\mathcal{L}$ is also weakly sequentially continuous. This proves that $\Psi$ is weakly sequentially continuous on $L^{p}\left(B_{R}\right)$. Since $\overline{\mathcal{F}(R)^{\omega}}$ is weakly sequentially compact, by Lemma 3 , it follows that $\Psi$ has a maximizer relative to $\overline{\mathcal{F}(R)^{\omega}}$, say $\tilde{\zeta}$. Fix $\zeta \in \overline{\mathcal{F}(R)^{\omega}}$, by convexity of $\overline{\mathcal{F}(R)^{\omega}}$, see Lemma 3 , it follows that for any $t \in[0,1], \tilde{\zeta}+t(\zeta-\tilde{\zeta}) \in$ $\overline{\mathcal{F}(R)^{\omega}}$. Next using the first variation of $\Psi$ at $\tilde{\zeta}$ we get

$$
\Psi\left(\tilde{\zeta}+t(\zeta-\tilde{\zeta})-\Psi(\tilde{\zeta})=t<\Psi^{\prime}(\tilde{\zeta}), \zeta-\tilde{\zeta}>+o(t)\right.
$$

as $t \rightarrow 0^{+}$; here $<,>$stands for the pairing between $L^{p}\left(B_{R}\right)$ and its dual, and $\Psi^{\prime}($. stands for the derivative. Since $\tilde{\zeta}$ is a maximizer we infer

$$
<\Psi^{\prime}(\tilde{\zeta}), \zeta-\tilde{\zeta}>\leq 0 .
$$

Therefore $\tilde{\zeta}$ is a maximizer for the linear functional $\left\langle\Psi^{\prime}(\tilde{\zeta}), .>\right.$, relative to $\overline{\mathcal{F}(R)^{\omega}}$. Since $\Psi^{\prime}(\tilde{\zeta})_{\tilde{\zeta}}$ can be identified with $K \tilde{\zeta}+\eta \in L^{p \prime}\left(B_{R}\right)$, it follows that $\tilde{\zeta}$ is a maximizer of $\int_{B_{R}} \zeta(K \tilde{\zeta}+\eta)$ relative to $\zeta \in \overline{\mathcal{F}(R)^{\omega}}$. From Lemma 2 we obtain

$$
-\Delta(K \tilde{\zeta}+\eta)=\tilde{\zeta}+h .
$$

Thus the level sets of $K \tilde{\zeta}+\eta$ on $\operatorname{supp}(\tilde{\zeta})$ are negligible, by [7, Lemma 7.7]. Whence we can apply Lemma 4 to deduce that $\tilde{\zeta} \in \mathcal{F}(R)$ and

$$
\tilde{\zeta}=\phi \circ(K \tilde{\zeta}+\eta)
$$

almost everywhere in $B_{R}$ for some increasing function $\phi$; in particular $\tilde{\zeta} \in \Sigma_{R}$. Now consider $\hat{\zeta}_{R} \in \Sigma_{R}$. Since $\Psi$ is weakly sequentially continuous, it follows that $\hat{\zeta}_{R}$ maximizes $\Psi$ relative to $\overline{\mathcal{F}(R)^{\omega}}$. Next by applying the first variation argument above we can similarly prove existence of an increasing function $\phi_{R}$ such that

$$
\hat{\zeta}_{R}=\phi_{R} \circ\left(K \hat{\zeta}_{R}+\eta\right),
$$

almost everywhere in $B_{R}$.

Lemma 6. Let $\gamma=\int_{\partial \mathbb{R}_{+}^{3}} h$. Then $\gamma>4 \pi c r_{h}$.

Proof. By the hypotheses on $r_{h}$ and $h$, that's (1) and (2), we have

$$
\gamma=\iint_{x_{1}^{2}+x_{2}^{2} \leq r_{h}^{2}} h\left(x_{1}, x_{2}\right) d x_{1} d x_{2} \geq c \iint_{x_{1}^{2}+x_{2}^{2} \leq r_{h}^{2}} \ln \left|x_{1} x_{2}\right| d x_{1} d x_{2}
$$




$$
\begin{gathered}
=c \int_{0}^{2 \pi} \int_{0}^{r_{h}} r \ln r^{2}|\sin \theta \cos \theta| d r d \theta \\
=c \int_{0}^{2 \pi} r_{h}^{2} \ln r_{h}-\frac{1}{2} r_{h}^{2}+\frac{1}{2} r_{h}^{2}(-\ln 2+\ln |\sin 2 \theta|) d \theta \\
=2 c \pi r_{h}^{2}\left(\ln r_{h}-\frac{1}{2}\right)-c \pi r_{h}^{2} \ln 2+\frac{c}{2} r_{h}^{2} \int_{0}^{2 \pi} \ln |\sin 2 \theta| d \theta \\
=2 c \pi r_{h}^{2} \ln \frac{r_{h}}{\sqrt{2 e}}+\frac{c}{2} r_{h}^{2} \int_{0}^{2 \pi} \ln |\sin \theta| d \theta=2 c \pi r_{h}^{2} \ln \frac{r_{h}}{\sqrt{2 e}} \\
+\frac{c}{2} r_{h}^{2}\left(\int_{0}^{\frac{\pi}{2}} \ln \sin \theta d \theta+\int_{\frac{\pi}{2}}^{\pi} \ln \sin \theta d \theta+\int_{\pi}^{\frac{3 \pi}{2}} \ln (-\sin \theta) d \theta+\int_{\frac{3 \pi}{2}}^{2 \pi} \ln (-\sin \theta) d \theta\right) \\
=2 c \pi r_{h}^{2} \ln \frac{r_{h}}{\sqrt{2 e}}+\frac{c}{2} r_{h}^{2}\left(4 \int_{0}^{\frac{\pi}{2}} \ln \sin \theta d \theta\right)=2 c \pi r_{h}^{2} \ln \frac{r_{h}}{\sqrt{2 e}} \\
+\frac{c}{2} r_{h}^{2}(-2 \pi \ln 2)=2 c \pi r_{h}^{2} \ln \frac{r_{h}}{2 \sqrt{e}}>4 \pi c r_{h} .
\end{gathered}
$$

Lemma 7. Let $R>r_{h}$ and $\hat{\zeta}_{R} \in \Sigma_{R}$, then $\operatorname{supp}\left(\hat{\zeta}_{R}\right) \subseteq B_{r_{h}}$, modulo a set of zero measure.

Proof. Suppose the assertion is false. Then there exist sequences $\left\{R_{n}\right\},\left\{x_{n}\right\}$ and $\left\{\hat{\zeta}_{R_{n}}\right\}:=\left\{\hat{\zeta}_{n}\right\}$ such that

(1) $R_{n} \rightarrow \infty$

(2) $\hat{\zeta}_{n} \in \Sigma_{R_{n}}$

(3) $x_{n} \in \operatorname{den}\left(\operatorname{supp}\left(\hat{\zeta}_{n}\right)\right)$ and $\left\|x_{n}\right\|_{\mathbb{R}_{+}^{3}} \rightarrow \infty$, where $\|\cdot\|_{\mathbb{R}_{+}^{3}}$ denote the usual Euclidean norm in $\mathbb{R}_{+}^{3}$. Without loss of generality we may assume that $\left\|x_{n}\right\|_{\mathbb{R}_{+}^{3}}=R_{n}$ and $\left\{R_{n}\right\}$ is increasing; moreover we may assume that $R_{n}>r_{h}$. Let us set $\psi_{n}:=K \hat{\zeta}_{n}+\eta$, and estimate $K \hat{\zeta}_{n}\left(x_{n}\right)$ :

$$
\begin{gathered}
K \hat{\zeta}_{n}\left(x_{n}\right) \leq \frac{1}{2 \pi} \int_{\mathbb{R}_{+}^{3}} \frac{1}{\left|x_{n}-y\right|} \hat{\zeta}_{n}(y) d y=\frac{1}{2 \pi} \int_{B_{r_{h}}} \frac{1}{\left|x_{n}-y\right|} \hat{\zeta}_{n}(y) d y+ \\
\quad \frac{1}{2 \pi} \int_{\mathbb{R}_{+}^{3}-B_{r_{h}}} \frac{1}{\left|x_{n}-y\right|} \hat{\zeta}_{n}(y) d y \leq \frac{1}{2 \pi}\left\|\hat{\zeta}_{n}\right\|_{1, B_{r_{h}}} \frac{1}{R_{n}-r_{h}}+c .
\end{gathered}
$$

Now we estimate $\eta\left(x_{n}\right)$ :

$$
\eta\left(x_{n}\right)=\frac{1}{2 \pi} \int_{\partial \mathbb{R}_{+}^{3}} \frac{1}{\left|x_{n}-y\right|} h(y) d \sigma(y) \leq \frac{\gamma}{2 \pi} \frac{1}{R_{n}-r_{h}} .
$$


From (11) and (12) we obtain

$$
\psi_{n}\left(x_{n}\right) \leq c+\frac{1}{2 \pi}\left\|\hat{\zeta}_{n}\right\|_{1, B_{r_{h}}} \frac{1}{R_{n}-r_{h}}+\frac{\gamma}{2 \pi} \frac{1}{R_{n}-r_{h}} .
$$

Notice that since $r_{h}>a$, we can find a sequence $\left\{y_{n}\right\}$ in $B_{r_{h}}$ such that $y_{n} \notin$ $\operatorname{den}\left(\operatorname{supp}\left(\hat{\zeta}_{n}\right)\right)$. Now, we estimate $\psi_{n}\left(y_{n}\right)$ from below.

$$
\begin{gathered}
K \hat{\zeta}_{n}\left(y_{n}\right) \geq \frac{1}{4 \pi} \int_{\mathbb{R}_{+}^{3}} \frac{1}{\left|y_{n}-y\right|} \hat{\zeta}_{n}(y) d y=\frac{1}{4 \pi} \int_{B_{r_{h}}} \frac{1}{\left|y_{n}-y\right|} \hat{\zeta}_{n}(y) d y+ \\
\frac{1}{4 \pi} \int_{\mathbb{R}_{+}^{3}-B_{r_{h}}} \frac{1}{\left|y_{n}-y\right|} \hat{\zeta}_{n}(y) d y \geq \frac{1}{4 \pi}\left\|\hat{\zeta}_{n}\right\|_{1, B_{r_{h}}} \frac{1}{2 r_{h}}+ \\
\frac{1}{4 \pi}\left(\left\|\zeta_{0}\right\|_{1}-\left\|\hat{\zeta}_{n}\right\|_{1, B_{r_{h}}}\right) \frac{1}{R_{n}+r_{h}} .
\end{gathered}
$$

Also,

$$
\eta\left(y_{n}\right)=\frac{1}{2 \pi} \int_{\partial \mathbb{R}_{+}^{3}} \frac{1}{\left|y_{n}-y\right|} h(y) d \sigma(y) \geq \frac{\gamma}{2 \pi} \frac{1}{2 r_{h}} .
$$

Therefore, from (14) and (15), we have

$$
\begin{gathered}
\psi_{n}\left(y_{n}\right) \geq \frac{1}{4 \pi}\left\|\hat{\zeta}_{n}\right\|_{1, B_{r_{h}}} \frac{1}{2 r_{h}}+ \\
\frac{1}{4 \pi}\left(\left\|\zeta_{0}\right\|_{1}-\left\|\hat{\zeta}_{n}\right\|_{1, B_{r_{h}}}\right) \frac{1}{R_{n}+r_{h}}+\frac{\gamma}{2 \pi} \frac{1}{2 r_{h}} .
\end{gathered}
$$

Therefore, from (13)and (16) we drive

$$
\begin{gathered}
\psi_{n}\left(x_{n}\right)-\psi_{n}\left(y_{n}\right) \leq c+\frac{1}{2 \pi}\left\|\hat{\zeta}_{n}\right\|_{1, B_{r_{h}}} \frac{1}{R_{n}-r_{h}}+\frac{\gamma}{2 \pi} \frac{1}{R_{n}-r_{h}} \\
-\frac{1}{4 \pi}\left\|\hat{\zeta}_{n}\right\|_{1, B_{r_{h}}} \frac{1}{2 r_{h}}-\frac{1}{4 \pi}\left(\left\|\zeta_{0}\right\|_{1}-\left\|\hat{\zeta}_{n}\right\|_{1, B_{r_{h}}}\right) \frac{1}{R_{n}+r_{h}}-\frac{\gamma}{2 \pi} \frac{1}{2 r_{h}} .
\end{gathered}
$$

Thus

$$
\limsup _{n \rightarrow \infty}\left(\psi_{n}\left(x_{n}\right)-\psi_{n}\left(y_{n}\right)\right) \leq c-\frac{\gamma}{4 \pi r_{h}}<0 .
$$

Where in the last inequality we have used Lemma 6. From (17)we infer existence of $n_{0} \in \mathbb{N}$ for which

$$
\psi_{n_{0}}\left(x_{n_{0}}\right)-\psi_{n_{0}}\left(y_{n_{0}}\right)<0 .
$$

However, from Lemma 4, and the Remark following it, there exists $\phi_{n_{0}}$, an increasing function, such that

$$
\hat{\zeta}_{n_{0}}=\phi_{n_{0}} \circ \psi_{n_{0}}
$$

everywhere in $B_{R_{n_{0}}}$. Therefore, $\psi_{n_{0}}$ attains its largest values over $\operatorname{den}\left(\operatorname{supp}\left(\hat{\zeta}_{n_{0}}\right)\right)$, so (18) is false. Hence we are done. $\square$

Remark. From Lemma 7 it readily follows that $\Sigma=\Sigma_{r_{h}}$. 
4. Proof of Theorem. The existence part of the Theorem follows from Lemma 7 and the remark following it. Now consider $\hat{\zeta} \in \Sigma$. Since $\hat{\zeta} \in \Sigma_{r_{h}}$, it follows that

$$
\hat{\zeta}=\phi_{r_{h}} \circ \psi,
$$

almost everywhere in $B_{r_{h}}$, where $\psi=K \hat{\zeta}+\eta$, thanks to Lemma 5 . Note that to derive (3) we only need to modify $\phi_{r_{h}}$ in order to have a similar functional equation as (19) to hold throughout $\mathbb{R}_{+}^{3}$. Since $\phi_{r_{h}}$ is an increasing function, we obtain

$$
\operatorname{supp}(\hat{\zeta})=\left\{x \in B_{r_{h}}: \psi \geq \lambda\right\},
$$

modulo a set of zero measure, where $\lambda$ is a positive constant. On the other hand for $|x| \geq 2 r_{h}$, we derive the following estimate

$$
\psi(x) \leq \frac{\|\hat{\zeta}\|_{1}+\gamma}{\pi|x|} .
$$

Thus, there exists $R^{\prime}>r_{h}$ such that

$$
\psi(z)<\frac{\lambda}{2},
$$

provided $z \in \mathbb{R}_{+}^{3}-B_{R^{\prime}}$. Finally, since $\hat{\zeta} \in \Sigma_{R^{\prime}}$ we can apply Lemma 5 once again to deduce the existence of another increasing function, say $\phi^{\prime}$, such that

$$
\hat{\zeta}=\phi^{\prime} \circ \psi,
$$

almost everywhere in $B_{R^{\prime}}$. We now define

$$
\phi(t)= \begin{cases}\phi^{\prime}(t) & \text { if } t \geq \lambda \\ 0 & \text { if } t<\lambda\end{cases}
$$

Therefore by applying (20), (21) and (22) we obtain $\hat{\zeta}=\phi \circ \psi$, almost everywhere in $\mathbb{R}_{+}^{3}$, as desired. Now using Lemma 2 and the fact that

$$
-\Delta \eta=h,
$$

almost everywhere in $\mathbb{R}_{+}^{3}$, we derive (3). This completes the proof of the Theorem.

\section{REFERENCES}

[1] R. A. Adams, Sobolev spaces, Academic, 1975.

[2] S. Agmon, The $L^{p}$ approach to the Dirichlet Problem, Ann. Scuola Norm. Pisa Cl. Sci., 13 (1959), pp. 405-448.

[3] T. B. BENJAMIN, The alliance of practical and analytical insights into the nonlinear problems of fluid mechanics, "Applications of methods of functional analysis to problems in mechanics", Lecture Notes in Mathematics, 503, Springer-Verlag, Berlin, (1976), pp. 8-29.

[4] G. R. Burton, Rearrangements of functions, maximization of convex functionals and vortex rings, Math. Ann., 276 (1987), pp. 225-253.

[5] G. R. BuRTon, Variational problems on classes of rearrangements and multiple configurations for steady vortices, Ann. Inst. H. Poincaré Anal. Non Lineáire, 6 (1989), pp. 295-319.

[6] B. Emamizadeh, F. Bahrami and M. H. Mehrabi, Steiner symmetric vortices attached to seamounts, Comm. Pure. Appl. Anal., 3:4 (2004), pp. 663-674.

[7] D. Gilbarg And N. Trudinger, Elliptic partial differential equations of second order, Springer, 1977.

[8] G. H. Hardy, J. E. Littlewood and G. Pólya, Inequalities, Cambridge: Cambridge University Press, 1934.

[9] J. Nycander and B. Emamizadeh, Variational problem for vortices attached to seamounts, Nonlinear Anal., 55 (2003), pp. 15-24. 
B. EMAMIZADEH AND M. H. MEHRABI 